\title{
Fluorine F-18 HX4
}

National Cancer Institute

\section{Source}

National Cancer Institute. Fluorine F-18 HX4. NCI Thesaurus. Code C74032.

A 2-nitroimidazole labeled with the positron-emitting radioisotope fluorine F18. HX4, the 2-nitroimidazole moiety of [F-18] HX4, is selectively bioreduced and bound in hypoxic tumor cells, permitting the imaging of hypoxic tumor cells with positron emission tomography (PET). 